\title{
Labeling Activity
}

National Cancer Institute

\section{Source}

National Cancer Institute. Labeling Activity. NCI Thesaurus. Code C84732.

The action of affixing an identifier to an item or the container for an item. 